\title{
Quaternionic bound states
}

\author{
Stefano De Leo ${ }^{1}$ and Gisele C Ducati ${ }^{2}$ \\ ${ }^{1}$ Department of Applied Mathematics, University of Campinas, PO Box 6065, SP 13083-970, \\ Campinas, Brazil \\ 2 Department of Mathematics, University of Parana PO Box 19081, PR 81531-970, Curitiba, \\ Brazil \\ E-mail: deleo@ime.unicamp.br and ducati@mat.ufpr.br
}

Received 13 October 2004, in final form 25 February 2005

Published 30 March 2005

Online at stacks.iop.org/JPhysA/38/3443

\begin{abstract}
We study the bound-state solutions of vanishing angular momentum in a quaternionic spherical square-well potential of finite depth. As in standard quantum mechanics, such solutions occur for discrete values of energy. At first glance, it seems that the continuity conditions impose a very restrictive constraint on the energy eigenvalues and, consequently, no bound states were expected for energy values below the pure quaternionic potential. Nevertheless, a careful analysis shows that pure quaternionic potentials do not remove bound states. It is also interesting to compare these new solutions with the bound state solutions of the trial-complex potential. The study presented in this paper represents a preliminary step towards a full understanding of the role that quaternionic potentials could play in quantum mechanics. Of particular interest for the authors is the analysis of confined wave packets and tunnelling times in this new formulation of quantum theory.
\end{abstract}

PACS numbers: $02.30 . \mathrm{Tb}, 03.65 . \mathrm{Ca}$

\section{Introduction}

In this introductory section, we briefly recall the basic kinematic and dynamical framework of quaternionic quantum mechanics. Obviously, it is not our purpose to give a complete introduction to the subject. For this and for further details on the mathematical formalism used in approaching the systematic development of quaternionic quantum mechanics, covering an axiomatic mathematical introduction to the quaternionic quantum field and an interesting discussion on the role that quaternions could play in searching for new physics, we refer the reader to the excellent book of Adler [1].

In previous years, the study of quaternionic quantum mechanics has shed new light on the mathematical structures used in quantum mechanics. In particular, a better understanding 
of the eigenvalue problem for real/complex and quaternionic linear differential operators $[2,3]$ allowed the solution of the Schrödinger equation in the presence of quaternionic potentials [4] without (unnecessary) complex translations [5, 6]. Nonetheless, quaternionic quantum mechanics is still in the early stage of development and what is still lacking is an interpretation of quaternionic potentials and proposals of experimental tests for quaternionic deviations from standard quantum mechanics. According to the nonrelativistic quaternionic scattering theory (developed in detail by Adler [1]) and the recent work on the quaternionic tunnelling effect [7], experiments to detect left/right transmission asymmetry in the presence of quaternionic barriers should involve $\mathrm{CP}$-violating (asymmetric) potentials. Consequently, the earliest proposals for tests of quaternionic quantum mechanics based on neutron-optical experiments gave (as expected by virtue of the study on quaternionic scattering theory) a null result [8-10]. It is thus important to look for other possible signatures of violation of the standard quantum mechanics due to quaternionic potentials. The main scope of this paper is to solve the quaternionic Schrödinger equation in the presence of binding potentials. This could represent a potential (and not yet explored) research field to determine if and where quaternionic potentials could be seen.

\section{Mathematical tools in quaternionic quantum mechanics}

To make our exposition self-contained and to facilitate access to the individual topics, the sections are rendered self-contained and, when necessary, we repeat the relevant material that recently appeared in the literature. Before beginning the study of quaternionic binding potentials, we summarize our notation and introduce some basic concepts of quaternionic quantum mechanics.

- Right multiplication by quaternionic scalars. In close analogy with the familiar complex quantum mechanics, the states of quaternionic quantum mechanics will be described by wavefunctions belonging to an abstract vector space. Due to the noncommutative nature of the quaternionic multiplication, we have to specify whether the quaternionic Hilbert space, $\mathcal{V}_{\mathbb{H}}$, is linear under right or left multiplication by quaternionic scalars. Following the Adler book [1], we adopt the convention of linearity from the right for the quaternionic Hilbert space. Thus, if $\Phi_{\mathbb{H}, 1}(\boldsymbol{r}, t)$ and $\Phi_{\mathbb{H}, 2}(\boldsymbol{r}, t)$ belong to $\mathcal{V}_{\mathbb{H}}$ and $q_{1}$ and $q_{2}$ are quaternionic scalars, then

$$
\Phi_{\mathbb{H}, 1}(\boldsymbol{r}, t) q_{1}+\Phi_{\mathbb{H}, 2}(\boldsymbol{r}, t) q_{2} \in \mathcal{V}_{\mathbb{H}} .
$$

- Anti-self-adjoint operators and the Schrödinger equation. The time evolution of $\Phi_{\mathbb{H}}(\boldsymbol{r}, t)$ is governed by the quaternionic Schrödinger equation

$$
\partial_{t} \Phi_{\mathbb{H}}(\boldsymbol{r}, t)=\mathcal{A}_{\mathbb{H}}(\boldsymbol{r}, t) \Phi_{\mathbb{H}}(\boldsymbol{r}, t),
$$

where

$$
\begin{aligned}
\mathcal{A}_{\mathbb{H}}(\boldsymbol{r}, t) & =\left[i \frac{\hbar^{2}}{2 m} \nabla^{2}-i V_{\mathbb{R}, 1}(\boldsymbol{r}, t)-j V_{\mathbb{R}, 2}(\boldsymbol{r}, t)-k V_{\mathbb{R}, 3}(\boldsymbol{r}, t)\right] / \hbar \\
& =\mathcal{A}_{\mathbb{C}}(\boldsymbol{r}, t)+j \mathcal{B}_{\mathbb{C}}(\boldsymbol{r}, t),
\end{aligned}
$$

is the anti-self-adjoint operator associated with the total energy of the system. The introduction of a pure quaternionic potential, $j \mathcal{B}_{\mathbb{C}}(r, t)$, does not modify the transition probability. There is an important difference between complex and quaternionic quantum mechanics. In complex quantum mechanics, we can trivially relate any anti-self-adjoint operator $\mathcal{A}_{\mathbb{C}}(\boldsymbol{r}, t)$ to the Hamiltonian observable $\mathcal{H}_{\mathbb{C}}(\boldsymbol{r}, t)$ by the factor $i$, namely

$$
\mathcal{H}_{\mathbb{C}}(\boldsymbol{r}, t)=i \hbar \mathcal{A}_{\mathbb{C}}(\boldsymbol{r}, t) .
$$


This is obviously not true in quaternionic quantum mechanics. In fact, the left action of the complex imaginary unit $i$ on $j \mathcal{B}_{\mathbb{C}}(r, t)$ still gives a pure quaternionic potential, $k \mathcal{B}_{\mathbb{C}}(\boldsymbol{r}, t)$.

- Stationary state and eigenvalue problem. In the presence of time-independent potentials, the quaternionic Schrödinger equation (2) becomes

$$
\begin{aligned}
\partial_{t} \Phi_{\mathbb{H}}(\boldsymbol{r}, t) & =\mathcal{A}_{\mathbb{H}}(\boldsymbol{r}) \Phi_{\mathbb{H}}(\boldsymbol{r}, t) \\
& =\left[i \frac{\hbar^{2}}{2 m} \nabla^{2}-i V_{\mathbb{R}, 1}(\boldsymbol{r})-j V_{\mathbb{R}, 2}(\boldsymbol{r})-k V_{\mathbb{R}, 3}(\boldsymbol{r})\right] \Phi_{\mathbb{H}}(\boldsymbol{r}, t) / \hbar .
\end{aligned}
$$

The method of separation of variables is still applicable if we set

$$
\Phi_{\mathbb{H}}(\boldsymbol{r}, t)=\varphi_{\mathbb{H}}(\boldsymbol{r}) \chi_{\mathbb{H}}(t)
$$

Due to the linearity under right multiplication by quaternionic scalars of $\mathcal{A}_{\mathbb{H}}(\boldsymbol{r})$, the right position of the time-dependent quaternionic function $\chi_{\mathbb{H}}(t)$ plays a fundamental role in separating the initial differential equation into two equations containing respectively the time and space variables. Explicitly, we find

$\hbar \dot{\chi}_{\mathbb{H}}(t) \chi_{\mathbb{H}}^{-1}(t)=\varphi_{\mathbb{H}}^{-1}(\boldsymbol{r})\left[i \frac{\hbar^{2}}{2 m} \nabla^{2}-i V_{\mathbb{R}, 1}(\boldsymbol{r})-j V_{\mathbb{R}, 2}(\boldsymbol{r})-k V_{\mathbb{R}, 3}(\boldsymbol{r})\right] \varphi_{\mathbb{H}}(\boldsymbol{r})$.

This equality is only possible if each of these functions is a constant, which we shall set equal to $\lambda \in \mathbb{H}$. Thus, the right linearity of the quaternionic Hilbert space $\mathcal{V}_{\mathbb{H}}$ implies a right eigenvalue problem for the anti-self-adjoint operator $\mathcal{A}_{\mathbb{H}}(\boldsymbol{r})$,

$$
\mathcal{A}_{\mathbb{H}}(\boldsymbol{r}) \varphi_{\mathbb{H}}(\boldsymbol{r})=\varphi_{\mathbb{H}}(\boldsymbol{r}) \lambda / \hbar \text {. }
$$

The need to use right eigenvalue in physical problems can also be demonstrated by simple mathematical arguments [2].

- Number systems and amplitudes of probabilities. To give a satisfactory probability interpretation, amplitudes of probability must be defined in associative division algebras. Amplitudes of probabilities defined in non-division algebras fail to satisfy the requirement that in the absence of quantum interference effects, probability amplitude superposition should reduce to probability superposition. The associative law of multiplication (which fails for the octonions) is needed to satisfy the completeness formula and to guarantee that the Schrödinger anti-self-adjoint operator leaves invariant the inner product. The important point to note here is that we can still use vectors in Clifford algebraic or octonionic Hilbert space. Nevertheless, amplitudes of probability must be defined in $\mathbb{C}$ or $\mathbb{H}$. For example, we can formulate a consistent complexified quaternionic [11] or octonionic [12] quantum mechanics by adopting complex inner products [13], i.e. complex projections of complexified quaternionic or octonionic inner product. The use of complex inner product represents a fundamental tool in applying a Clifford algebra formalism to physics and plays a fundamental role in looking for geometric interpretation of the algebraic structures in relativistic equations [14-17] and gauge theories [18, 19]. The choice of quaternionic inner product seems to be best adapted to investigate deviations from the standard complex theory. In this paper, we shall work with quaternionic inner products. Thus, with each pair of elements of the quaternionic Hilbert space $\mathcal{V}_{\mathbb{H}}$, we associate a quaternionic inner product defined by

$$
\left\langle\varphi_{\mathbb{H}}, \psi_{\mathbb{H}}\right\rangle:=\int \mathrm{d} \boldsymbol{r} \bar{\varphi}_{\mathbb{H}}(\boldsymbol{r}) \psi_{\mathbb{H}}(\boldsymbol{r}) \in \mathbb{H},
$$

where $\bar{\varphi}_{\mathbb{H}}(\boldsymbol{r})$ denotes the quaternionic conjugate of

$$
\varphi_{\mathbb{H}}(\boldsymbol{r})=\varphi_{\mathbb{R}, 0}(\boldsymbol{r})+i \varphi_{\mathbb{R}, 1}(\boldsymbol{r})+j \varphi_{\mathbb{R}, 2}(\boldsymbol{r})+k \varphi_{\mathbb{R}, 3}(\boldsymbol{r}),
$$


i.e.

$$
\bar{\varphi}_{\mathbb{H}}(\boldsymbol{r})=\varphi_{\mathbb{R}, 0}(\boldsymbol{r})-i \varphi_{\mathbb{R}, 1}(\boldsymbol{r})-j \varphi_{\mathbb{R}, 2}(\boldsymbol{r})-k \varphi_{\mathbb{R}, 3}(\boldsymbol{r}) .
$$

- Energy eigenvalues. The anti-Hermiticity of $\mathcal{A}_{\mathbb{H}}(\boldsymbol{r})$,

$$
\left\langle\varphi_{\mathbb{H}}, \mathcal{A}_{\mathbb{H}} \psi_{\mathbb{H}}\right\rangle=-\left\langle\mathcal{A}_{\mathbb{H}} \varphi_{\mathbb{H}}, \psi_{\mathbb{H}}\right\rangle
$$

implies $\lambda=-\bar{\lambda}$. Consequently, observing that $\lambda$ is related to the total energy of the system and setting

$$
\lambda=-\left(i E_{1}+j E_{2}+k E_{3}\right),
$$

the quaternionic energy eigenvalue equation (4) becomes

$$
\mathcal{A}_{\mathbb{H}}(\boldsymbol{r}) \varphi_{\mathbb{H}}(\boldsymbol{r})=-\varphi_{\mathbb{H}}(\boldsymbol{r})\left(i E_{1}+j E_{2}+k E_{3}\right) / \hbar .
$$

In the complex limit, the previous equation reduces to

$$
\mathcal{H}_{\mathbb{C}}(\boldsymbol{r}) \varphi_{\mathbb{C}}(\boldsymbol{r})=i \hbar \mathcal{A}_{\mathbb{C}}(\boldsymbol{r}, t) \varphi_{\mathbb{C}}(\boldsymbol{r})=E_{1} \varphi_{\mathbb{C}}(\boldsymbol{r})
$$

Eigenvectors of anti-self-adjoint quaternionic operator divide into mutually orthogonal eigenclasses. Each eigenclass corresponds to a ray of physically equivalent states. Eigenvectors within each eigenclass are not orthogonal and have eigenvalues related by the automorphism transformation

$$
\bar{u}\left(i \lambda_{1}+j \lambda_{2}+k \lambda_{3}\right) u,
$$

with $u$ unitary quaternion. By choosing a particular automorphism transformation, i.e.

$$
u\left(\lambda_{1}, \lambda_{2}, \lambda_{3}\right)=\sqrt{\frac{\lambda_{1}+\sqrt{\lambda_{1}^{2}+\lambda_{2}^{2}+\lambda_{3}^{2}}}{2 \sqrt{\lambda_{1}^{2}+\lambda_{2}^{2}+\lambda_{3}^{2}}}}\left[1+j \frac{\lambda_{2}-i \lambda_{3}}{i\left(\lambda_{1}+\sqrt{\lambda_{1}^{2}+\lambda_{2}^{2}+\lambda_{3}^{2}}\right)}\right],
$$

we set a particular representative ray within each eigenclass whose eigenvalue is complex,

$$
\lambda=i \sqrt{\lambda_{1}^{2}+\lambda_{2}^{2}+\lambda_{3}^{2}} .
$$

Hence, within each eigenclass of $\mathcal{A}_{\mathbb{H}}(\boldsymbol{r})$ eigenvectors, there is a ray

$$
\Psi_{\mathbb{H}}(\boldsymbol{r})=\varphi_{\mathbb{H}}(\boldsymbol{r}) u
$$

for which

$$
\mathcal{A}_{\mathbb{H}}(\boldsymbol{r}) \Psi_{\mathbb{H}}(\boldsymbol{r})=-\Psi_{\mathbb{H}}(\boldsymbol{r}) i E / \hbar .
$$

Thus, for stationary states, the quaternionic Schrödinger equation can be rewritten as follows:

$$
\left[i \frac{\hbar^{2}}{2 m} \nabla^{2}-i V_{\mathbb{R}, 1}(\boldsymbol{r})-j V_{\mathbb{R}, 2}(\boldsymbol{r})-k V_{\mathbb{R}, 3}(\boldsymbol{r})\right] \Psi_{\mathbb{H}}(\boldsymbol{r})=-\Psi_{\mathbb{H}}(\boldsymbol{r}) i E .
$$

Before concluding this section, let us come back to the chosen automorphism transformation $u$ and consider the limit $\lambda_{2,3} \rightarrow 0$ (complex eigenvalue case, $i \lambda_{1}$ ). We have to distinguish between $\lambda_{1}=\left|\lambda_{1}\right|$ and $\lambda_{1}=-\left|\lambda_{1}\right|$. A simple calculation shows that

$$
\lim _{\lambda_{2,3} \rightarrow 0} u\left(\left|\lambda_{1}\right|, \lambda_{2}, \lambda_{3}\right)=1 .
$$

For the case $\lambda_{1}=-\left|\lambda_{1}\right|$ the situation is a little more complicated. For example, we find

$$
\begin{aligned}
& \lim _{\lambda_{2} \rightarrow 0}\left[\lim _{\lambda_{3} \rightarrow 0} u\left(-\left|\lambda_{1}\right|, \lambda_{2}, \lambda_{3}\right)\right]=k, \\
& \lim _{\lambda_{3} \rightarrow 0}\left[\lim _{\lambda_{2} \rightarrow 0} u\left(-\left|\lambda_{1}\right|, \lambda_{2}, \lambda_{3}\right)\right]=-j, \\
& \lim _{\lambda_{3} \rightarrow 0}\left[\lim _{\lambda_{2} \rightarrow \lambda_{3}} u\left(-\left|\lambda_{1}\right|, \lambda_{2}, \lambda_{3}\right)\right]=(k-j) / \sqrt{2} .
\end{aligned}
$$

This is due to the infinite possibilities that we have, in a three-dimensional space, to 
rotate the vector $\left(-\left|\lambda_{1}\right|, 0,0\right)$ into $\left(\left|\lambda_{1}\right|, 0,0\right)$. The given limits correspond respectively to rotations of an angle $\pi$ around the vectors $\boldsymbol{z}, \boldsymbol{y}$ and $\boldsymbol{y}+\boldsymbol{z}$.

\section{Spherically symmetric potentials}

The central force problem in which $V_{\mathbb{R}, 1}(r)=V_{\mathbb{R}, 1}(r)$ occupies a prominent place in (complex) quantum mechanics because many important applications involve potentials that are at least approximately spherically symmetric. In this section, we extend this symmetry to the quaternionic part of the potential,

$$
V_{\mathbb{R}, 2}(\boldsymbol{r})=V_{\mathbb{R}, 2}(r) \quad \text { and } \quad V_{\mathbb{R}, 3}(\boldsymbol{r})=V_{\mathbb{R}, 3}(r) .
$$

Since the quaternionic potential

$$
V_{\mathbb{H}}(r)=i V_{\mathbb{R}, 1}(r)+j V_{\mathbb{R}, 2}(r)+k V_{\mathbb{R}, 3}(r),
$$

depends only on the distance $r$ of the particle from the origin, spherical coordinates are best adapted to the problem. Thus, we express the Laplace operator in $\mathcal{A}_{\mathbb{H}}(\boldsymbol{r})$ in spherical coordinates $(r, \theta, \varphi)$ by the well-known formula

$$
\nabla^{2}=\frac{\partial^{2}}{\partial r^{2}}+\frac{2}{r} \frac{\partial}{\partial r}-\frac{L^{2}}{\hbar^{2} r^{2}}
$$

Since the operator $\boldsymbol{L}^{2}$ depends only on $\theta$ and $\varphi$, by using the (complex) spherical harmonic functions $Y_{\mathbb{C}}^{l m}(\theta, \varphi)$,

$$
\Psi_{\mathbb{H}}(\boldsymbol{r})=R_{\mathbb{H}}(r) Y_{\mathbb{C}}^{l m}(\theta, \varphi),
$$

we can reduce the Schrödinger equation (8) to the following quaternionic radial equation:

$\left\{i \frac{\hbar^{2}}{2 m}\left[\frac{\mathrm{d}^{2}}{\mathrm{~d} r^{2}}+\frac{2}{r} \frac{\mathrm{d}}{\mathrm{d} r}\right]-i V_{\mathbb{R}, 1}^{\mathrm{eff}}(r)-j V_{\mathbb{R}, 2}(r)-k V_{\mathbb{R}, 3}(r)\right\} R_{\mathbb{H}}(r)=-R_{\mathbb{H}}(r) i E$,

where

$$
V_{\mathbb{R}, 1}^{\mathrm{eff}}(r)=V_{\mathbb{R}, 1}(r)+\frac{l(l+1) \hbar^{2}}{2 m r^{2}} .
$$

By the change in the function

$$
R_{\mathbb{H}}(r)=U_{\mathbb{H}}(r) / r,
$$

we obtain for $U_{\mathbb{H}}(r)$ the following differential equation:

$$
\left[i \frac{\hbar^{2}}{2 m} \frac{\mathrm{d}^{2}}{\mathrm{~d} r^{2}}-i V_{\mathbb{R}, 1}^{\mathrm{eff}}(r)-j V_{\mathbb{R}, 2}(r)-k V_{\mathbb{R}, 3}(r)\right] U_{\mathbb{H}}(r)=-U_{\mathbb{H}}(r) i E .
$$

\subsection{Spherical potential trap and quaternionic eigenfunctions}

To find the energy levels of a particle with angular momentum $l=0$ in the presence of a quaternionic spherical potential trap

$$
V_{\mathbb{H}}(r)=\left\{\begin{array}{lll}
0 & r<a & (\text { REGION I), } \\
i V_{1}+j V_{2}+k V_{3} & r>a & \text { (REGION II) }
\end{array}\right.
$$

we substitute such a potential in equation (10). This yields the two ordinary differential equations

$$
i \ddot{U}_{\mathrm{I}, \mathbb{H}}(r)=-U_{\mathrm{I}, \mathbb{H}}(r) i 2 m E / \hbar^{2}
$$


and

$$
i \ddot{U}_{\mathrm{II}, \mathbb{H}}(r)-\left[2 m\left(i V_{1}+j V_{2}+k V_{3}\right) / \hbar^{2}\right] U_{\mathrm{II}, \mathbb{H}}(r)=-U_{\mathrm{II}, \mathbb{H}}(r) i 2 m E / \hbar^{2} .
$$

The solution in region I is easily obtained by observing that to the standard complex sine/cosine solution we have to add a pure quaternionic hyperbolic sine/cosine solution,

$$
U_{\mathrm{I}, \mathbb{H}}(r)=\sin (\epsilon r) \alpha_{1}+\cos (\epsilon r) \beta_{1}+j \sinh (\epsilon r) \gamma_{1}+j \cosh (\epsilon r) \delta_{1},
$$

where $\epsilon=\sqrt{2 m E / \hbar^{2}}$ and $\alpha_{1}, \beta_{1}, \gamma_{1}, \delta_{1}$ are constant complex coefficients to be determined by boundary conditions and continuity constraints. To guarantee the correct behaviour of the solution at the origin, the wavefunction must satisfy $U_{\mathrm{I}, \mathbb{H}}(0)=0$, thus we have to impose the following constraint $\beta_{1}=\delta_{1}=0$. Consequently, the solution inside the well $(r<a)$ is given by

$$
U_{\mathrm{I}, \mathbb{H}}(r)=\sin (\epsilon r) \alpha_{1}+j \sinh (\epsilon r) \gamma_{1} .
$$

The discussion in region II is a little more complicated (see appendix A for the explicit calculations) and provides the following solution:

$U_{\mathrm{II}, \mathbb{H}}(r)=(1+j w)\left\{\sinh \left[v_{-} r\right] \alpha_{2}+\cosh \left[v_{-} r\right] \beta_{2}\right\}+(z+j)\left\{\sinh \left[v_{+} r\right] \gamma_{2}+\cosh \left[v_{+} r\right] \delta_{2}\right\}$,

where

$\nu_{ \pm}=\sqrt{\frac{2 m}{\hbar^{2}}\left(V_{1} \pm \sqrt{E^{2}-V_{2}^{2}-V_{3}^{2}}\right)}, \quad w=-i \frac{V_{2}-i V_{3}}{E+\sqrt{E^{2}-V_{2}^{2}-V_{3}^{2}}}$

and

$z=i \frac{V_{2}+i V_{3}}{E+\sqrt{E^{2}-V_{2}^{2}-V_{3}^{2}}}$.

Observing that, independently of the value of $E$, the real part of $v_{+}$is never null, the condition that the wavefunction does not diverge at $r=+\infty$ requires a first constraint i.e. $\gamma_{2}=-\delta_{2}$ (without loss of generality we can choose $\operatorname{Re}\left[v_{ \pm}\right]>0$ ). Thus, the solution in region II can be rewritten as

$$
U_{\mathrm{II}, \mathbb{H}}(r)=(1+j w)\left[\sinh \left(v_{-} r\right) \alpha_{2}+\cosh \left(\nu_{-} r\right) \beta_{2}\right]+(z+j) \exp \left[-v_{+} r\right] \delta_{2} .
$$

If the total energy $E$ is greater than $\sqrt{V_{1}^{2}+V_{2}^{2}+V_{3}^{2}}$, we have the free particle case. In fact, for $E>\sqrt{V_{1}^{2}+V_{2}^{2}+V_{3}^{2}}, \nu_{-}$is a complex imaginary number and no constraint exists for the coefficient $\alpha_{2}$ and $\beta_{2}$. The continuity relations in $r=a$ for the wavefunctions and their gradients can be satisfied for all values of the energy and the energy levels are therefore not quantized.

The bound particle case corresponds to $E<\sqrt{V_{1}^{2}+V_{2}^{2}+V_{3}^{2}}$. In this case, the condition that the wavefunction does not diverge at $r=+\infty$ gives an additional constraint on the coefficients $\alpha_{2}$ and $\beta_{2}$, i.e. $\alpha_{2}=-\beta_{2}$. Thus, we have a spatially damped solution in region II given by

$U_{\mathrm{II}, \mathbb{H}}\left(r ; E<\sqrt{V_{1}^{2}+V_{2}^{2}+V_{3}^{2}}\right)=(1+j w) \exp \left[-v_{-} r\right] \beta_{2}+(z+j) \exp \left[-v_{+} r\right] \delta_{2}$.

It remains to adjust the coefficients $\alpha_{1}, \gamma_{1}, \beta_{2}$ and $\delta_{2}$ so that the amplitudes and gradients at $r=a$ are continuous. This will imply a quantization for the energy levels. 


\subsection{Quaternionic bound states}

The continuity conditions

$$
U_{\mathrm{I}, \mathbb{H}}(a)=U_{\mathrm{II}, \mathbb{H}}(a) \quad \text { and } \quad \dot{U}_{\mathrm{I}, \mathbb{H}}(a)=\dot{U}_{\mathrm{II}, \mathbb{H}}(a)
$$

imply

$$
\begin{aligned}
& \sin (\epsilon a) \alpha_{1}+j \sinh (\epsilon a) \gamma_{1}=\exp \left[-v_{-} a\right] \beta_{2}+z \exp \left[-v_{+} a\right] \delta_{2} \\
&+j\left[w \exp \left[-v_{-} a\right] \beta_{2}+\exp \left[-v_{+} a\right] \delta_{2}\right], \\
&-\epsilon\left[\cos (\epsilon a) \alpha_{1}\right.\left.+j \cosh (\epsilon a) \gamma_{1}\right]=v_{-} \exp \left[-v_{-} a\right] \beta_{2}+z v_{+} \exp \left[-v_{+} a\right] \delta_{2} \\
&+j\left[w v_{-} \exp \left[-v_{-} a\right] \beta_{2}+v_{+} \exp \left[-v_{+} a\right] \delta_{2}\right] .
\end{aligned}
$$

Separating the complex from the pure quaternionic part in the previous equations and eliminating the coefficients $\alpha_{1}$ and $\gamma_{1}$, we obtain the following matrix equation:

$$
\left(\begin{array}{cc}
v_{-} \tan (\epsilon a)+\epsilon & z\left[v_{+} \tan (\epsilon a)+\epsilon\right] \\
w\left[v_{-} \tanh (\epsilon a)+\epsilon\right] & v_{+} \tanh (\epsilon a)+\epsilon
\end{array}\right)\left(\begin{array}{c}
\exp \left[-v_{-} a\right] \beta_{2} \\
\exp \left[-v_{+} a\right] \delta_{2}
\end{array}\right)=0 .
$$

This matrix equation has a non-trivial solution only if the determinant of

$$
\left(\begin{array}{cr}
v_{-} \tan (\epsilon a)+\epsilon & z\left[v_{+} \tan (\epsilon a)+\epsilon\right] \\
w\left[v_{-} \tanh (\epsilon a)+\epsilon\right] & v_{+} \tanh (\epsilon a)+\epsilon
\end{array}\right)
$$

vanishes, i.e.

$$
z w=\frac{\left[v_{-} \tan (\epsilon a)+\epsilon\right]\left[v_{+} \tanh (\epsilon a)+\epsilon\right]}{\left[v_{-} \tanh (\epsilon a)+\epsilon\right]\left[v_{+} \tan (\epsilon a)+\epsilon\right]}
$$

This equation can be rewritten in a more convenient form, i.e.

$$
\begin{aligned}
& \tan (\epsilon a)=-\frac{\epsilon}{v_{-}} \frac{\left(v_{+}-z w v_{-}\right) \tanh (\epsilon a)+(1-z w) \epsilon}{\epsilon} \\
& =-\frac{\sqrt{v_{c}^{2}-\sqrt{\epsilon^{4}-v_{q}^{4}}}}{\left.\sqrt{v_{c}^{2}+\sqrt{\epsilon^{4}-v_{q}^{4}}}-\frac{v_{q}^{4}}{\epsilon^{2}+\sqrt{\epsilon^{4}-v_{q}^{4}}} \sqrt{v_{c}^{2}-\sqrt{\epsilon^{4}-v_{q}^{4}}}\right] \tanh (\epsilon a)+\left[1-\frac{v_{q}^{4}}{\epsilon^{2}+\sqrt{\epsilon^{4}-v_{q}^{4}}}\right] \epsilon} \\
& \times \frac{\sqrt{v_{c}^{2}+\sqrt{\epsilon^{4}-v_{q}^{4}}}\left[1-\frac{v_{q}^{4}}{\epsilon^{2}+\sqrt{\epsilon^{4}-v_{q}^{4}}}\right] \tanh (\epsilon a)+\left[1-\frac{v_{q}^{4}}{\left(\epsilon^{2}+\sqrt{\epsilon^{4}-v_{q}^{4}}\right)^{2}} \sqrt{\frac{v_{c}^{2}+\sqrt{\epsilon^{4}-v_{q}^{4}}}{v_{c}^{2}-\sqrt{\epsilon^{4}-v_{q}^{4}}}}\right] \epsilon}{=} \\
& f\left(\epsilon ; v_{c}, v_{q}\right),
\end{aligned}
$$

where

$$
v_{c}=\sqrt{2 m V_{1} / \hbar^{2}} \text { and } v_{q}=\sqrt{2 m \sqrt{V_{2}^{2}+V_{3}^{2}} / \hbar^{2}}
$$

Let us first examine the case: $\sqrt{V_{2}^{2}+V_{3}^{2}}<E<\sqrt{V_{1}^{2}+V_{2}^{2}+V_{3}^{2}}$. Observing that this condition on the energy eigenvalues implies $v_{q}<\epsilon$ and $\nu_{c}>\left(\epsilon^{4}-v_{q}^{4}\right)^{1 / 4}$, it can be immediately seen that equation (17) represents a real equation which generalizes (to the 


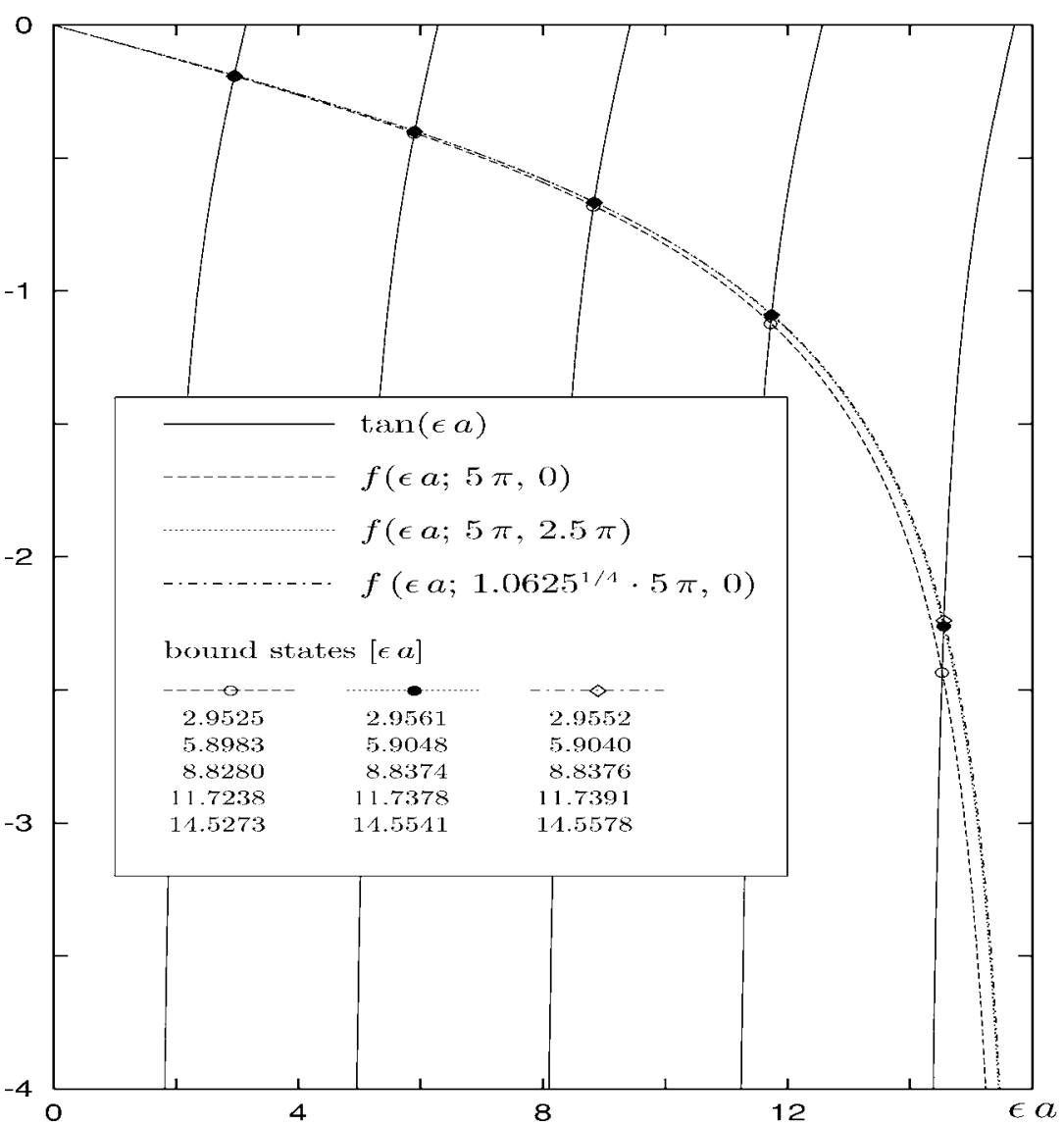

Figure 1. Graphical solutions of equation (17). The circle, bullet and diamond symbols respectively, represent the vanishing angular momentum bound state solutions of the radial Schrödinger equation for a complex $\left(i V_{1}\right)$, quaternionic $\left(i V_{1}+j V_{2}+k V_{3}\right)$, and trial-complex $\left(i \sqrt{V_{1}^{2}+V_{2}^{2}+V_{3}^{2}}\right)$ square-well potential. For the convenience of the reader we also list the numerical values of the bound energies. The plots refer to the following potentials: $a \sqrt{2 m V_{1} / \hbar^{2}}=5 \pi$ and $\sqrt{2 m \sqrt{V_{2}^{2}+V_{3}^{2}} / \hbar^{2}}=2.5 \pi$.

'quaternionic' case) the well-known equation obtained in 'complex' quantum mechanics, i.e.

$$
\begin{aligned}
\tan (\epsilon a) & =f\left(\epsilon ; v_{c}, 0\right) \\
& =-\frac{\epsilon}{\sqrt{v_{c}^{2}-\epsilon^{2}}} .
\end{aligned}
$$

One may ask whether this is still true for energy values below $\sqrt{V_{2}^{2}+V_{3}^{2}}$. For $E<\sqrt{V_{2}^{2}+V_{3}^{2}}$; it can be immediately seen that $\nu_{ \pm}$and $z w$ are complex and this seems to be too restrictive. Consequently, no bound states should be expected for $E<\sqrt{V_{2}^{2}+V_{3}^{2}}$. Nevertheless, by simple algebraic manipulations (see appendix B for the explicit calculations), it can be shown that equation (17) still represents a real equation and bound states could exist below the pure quaternionic potential. Graphical solutions of equation (17) give the energies of the bound states of a particle in a quaternionic spherically symmetric potential trap, see figures 1 and 2 . It is also instructive to compare the quaternionic bound states with the bound states of the trial-complex potential $\sqrt{V_{1}^{2}+V_{2}^{2}+V_{3}^{2}}$. 


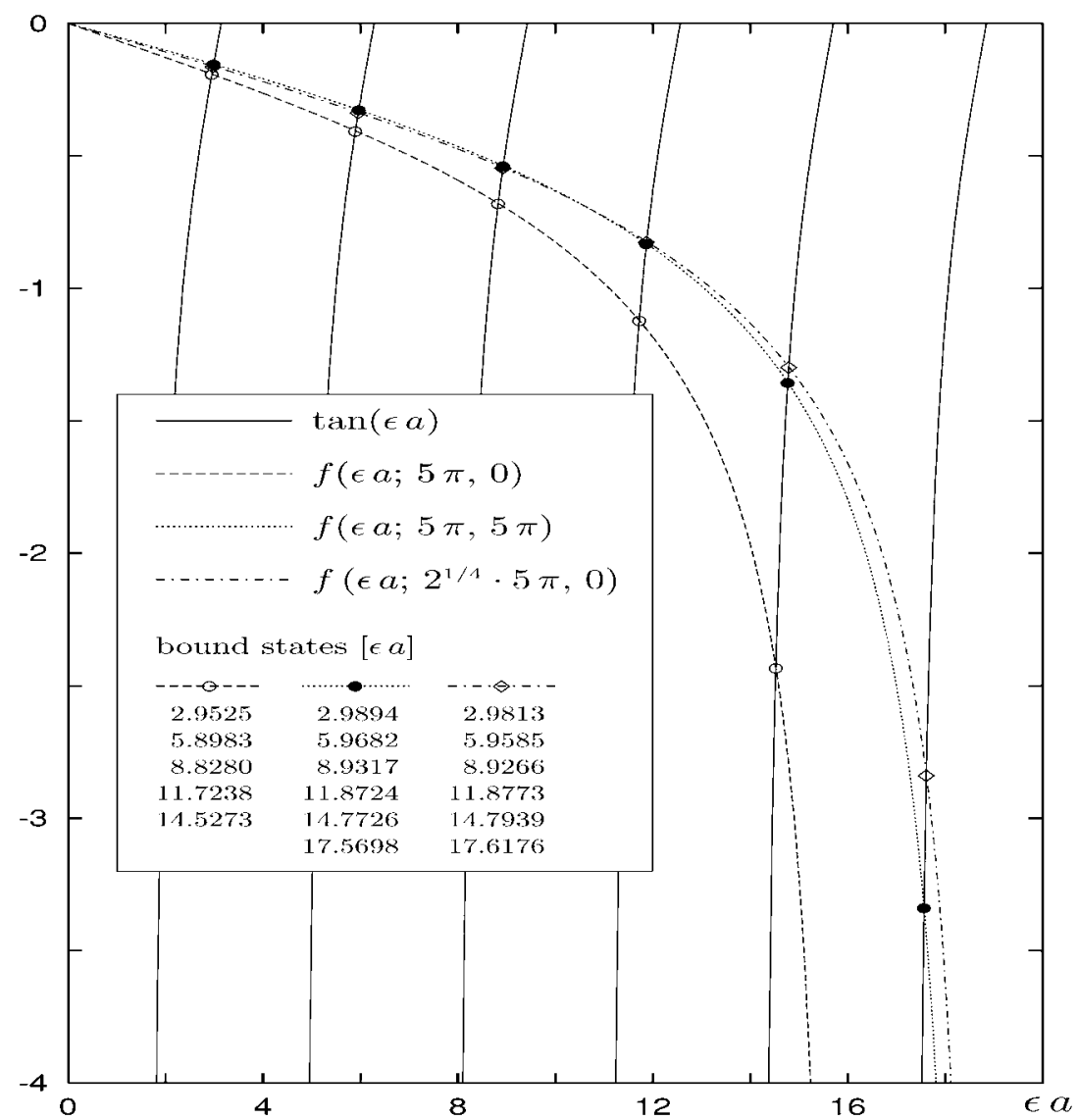

Figure 2. Graphical solutions of equation (17). The circle, bullet and diamond symbols respectively, represent the vanishing angular momentum bound state solutions of the radial Schrödinger equation for a complex $\left(i V_{1}\right)$, quaternionic $\left(i V_{1}+j V_{2}+k V_{3}\right)$ and trial-complex $\left(i \sqrt{V_{1}^{2}+V_{2}^{2}+V_{3}^{2}}\right.$ ) square-well potential. For the convenience of the reader we also list the numerical values of the bound energies. The plots refer to the following potentials: $a \sqrt{2 m V_{1} / \hbar^{2}}=5 \pi$ and $\sqrt{2 m \sqrt{V_{2}^{2}+V_{3}^{2}} / \hbar^{2}}=5 \pi$.

\section{Conclusions}

This work was intended as an attempt to motivate the study of quaternionic formulations of quantum mechanics and to look for deviations from the standard complex theory. The analysis presented in this paper represents a preliminary discussion on quaternionic bound states and touched only a few aspects of the theory. In particular, no attempt has been made here to develop a quaternionic perturbation theory, to treat time-dependent potentials $[1,21]$ or to study the behaviour of confined quaternionic wave packets [22, 23]. These topics exceed the scope of this paper but surely represent important points to be investigated in view of a complete understanding of how and where quaternionic potentials could be seen and if they really exist in nature.

Since the quaternions can be represented by $2 \times 2$ complex matrices, it is obvious that our quaternionic analysis in region I and II can be 'translated' into a problem involving $4 \times 4$ complex matrices. Such a complex problem seems to be very close to the square-well potential 
case for the standard Dirac equation. Thus, an interesting investigation could be the study of the difference between the four complex components of the 'translated' quaternionic spinor (obtained by solving the Schrödinger equation in the presence of a quaternionic square-well potential) and of the Dirac spinor (obtained by solving the Dirac equation in the presence of a complex square-well potential).

\section{Acknowledgments}

The authors wish to express their gratitude to an anonymous referee for several helpful suggestions on the mathematical presentation of the subject and for his active role in drawing attention to stimulating unsolved questions to be investigated in the future.

\section{Appendix A}

To calculate the radial solution in region II, we have to solve a second-order differential equation with quaternionic constant coefficients,

$$
\left\{i \frac{\hbar^{2}}{2 m} \frac{\mathrm{d}^{2}}{\mathrm{~d} r^{2}}-i V_{1}-j V_{2}-k V_{3}\right\} U_{\mathrm{II}, \mathbb{H}}(r)=-U_{\mathrm{II}, \mathbb{H}}(r) i E .
$$

We refer the reader to $[4,20]$ for a detailed analysis of quaternionic differential operators. In this appendix, we will touch only a few aspect of the theory, by restricting our attention to differential operators with quaternionic constant coefficients. A (right-complex linear) solution of equation (A.1) can be written in terms of (left-acting) quaternionic $(q)$ and complex $(v)$ coefficients, i.e.

$$
q \exp [v r] \text {. }
$$

To determine these coefficients, let us apply to equation (A.1) the anti-Hermitian operator

In this way we obtain

$$
i \frac{\hbar^{2}}{2 m} \frac{\mathrm{d}^{2}}{\mathrm{~d} r^{2}}-i V_{1}-j V_{2}-k V_{3}
$$

$$
\left\{-\left(\frac{\hbar^{2}}{2 m}\right)^{2} \frac{\mathrm{d}^{4}}{\mathrm{~d} r^{4}}+2 \frac{\hbar^{2}}{2 m} V_{1} \frac{\mathrm{d}^{2}}{\mathrm{~d} r^{2}}-\left(V_{1}^{2}+V_{2}^{2}+V_{3}^{2}\right)\right\} U_{\mathrm{II}, \mathbb{H}}(r)=-E^{2} U_{\mathrm{II}, \mathbb{H}}(r),
$$

which represents a real differential equation. Consequently, the quaternionic factor $q$ can be factorized and the complex coefficient $v$ is calculated once the following algebraic equation is solved:

$$
\left(\frac{\hbar^{2}}{2 m}\right)^{2} v^{4}-2 \frac{\hbar^{2}}{2 m} V_{1} v^{2}+V_{1}^{2}+V_{2}^{2}+V_{3}^{2}-E^{2}=0 .
$$

The solutions for the complex coefficients $v$ are given by

$$
v_{1,2}= \pm v_{-} \quad \text { and } \quad v_{3,4}= \pm v_{+} \quad \text { where } \quad v_{ \pm}=\sqrt{\frac{2 m}{\hbar^{2}}\left(V_{1} \pm \sqrt{E^{2}-V_{2}^{2}-V_{3}^{2}}\right)}
$$

As suggested by an anonymous referee, an alternative way to obtain equation (A.3) is to substitute $q \exp [v r]$ in equation (A.1) and multiply the new equation by $-i$ from the left and by $i$ from the right,

$$
\begin{aligned}
& q \frac{\hbar^{2}}{2 m} v^{2}+i\left(i V_{1}+j V_{2}+k V_{3}\right) q=i q i E, \\
& i q i \frac{\hbar^{2}}{2 m} v^{2}+\left(i V_{1}+j V_{2}+k V_{3}\right) i(i q i)=q E .
\end{aligned}
$$

These equations give, after eliminating $i q i$ in favour of $q$, equation (A.3) for $v$. 
Coming back to equation (A.1), for $E \neq \sqrt{V_{2}^{2}+V_{3}^{2}}$ and $E \neq \sqrt{V_{1}^{2}+V_{2}^{2}+V_{3}^{2}}$ we then find four (right-complex) linear-independent solutions [4], i.e.

$q_{-} \exp \left[-v_{-} r\right], \quad q_{-} \exp \left[v_{-} r\right], \quad q_{+} \exp \left[-v_{+} r\right], \quad$ and $q_{+} \exp \left[v_{+} r\right]$.

To find the quaternionic factor, $q_{-}$(associated with $\left.\exp \left[ \pm v_{-} r\right]\right)$ we set $q_{-}=1+j w(w \in \mathbb{C})$. This choice is possible due to the right-complex linearity of the quaternionic differential equation (A.1). It follows immediately that

$i(1+j w)\left(V_{1}-\sqrt{E^{2}-V_{2}^{2}-V_{3}^{2}}\right)-\left(i V_{1}+j V_{2}+k V_{3}\right)(1+j w)=-(1+j w) i E$.

It is easy to check that the previous equation implies

$$
\begin{aligned}
& i\left(E-\sqrt{E^{2}-V_{2}^{2}-V_{3}^{2}}\right)+\left(V_{2}+i V_{3}\right) w=0, \\
& i\left(E+\sqrt{E^{2}-V_{2}^{2}-V_{3}^{2}}\right) w-\left(V_{2}-i V_{3}\right)=0 .
\end{aligned}
$$

Finally,

$$
w=-i \frac{E-\sqrt{E^{2}-V_{2}^{2}-V_{3}^{2}}}{V_{2}+i V_{3}}=-i \frac{V_{2}-i V_{3}}{E+\sqrt{E^{2}-V_{2}^{2}-V_{3}^{2}}} .
$$

To find the quaternionic factor, $q_{+}$(associated with $\left.\exp \left[ \pm v_{+} r\right]\right)$ we choose $q_{+}=z+j(z \in \mathbb{C})$. A calculation similar to previous one gives

$$
z=i \frac{E-\sqrt{E^{2}-V_{2}^{2}-V_{3}^{2}}}{V_{2}-i V_{3}}=i \frac{V_{2}+i V_{3}}{E+\sqrt{E^{2}-V_{2}^{2}-V_{3}^{2}}}
$$

\section{Appendix B}

In this appendix, we are interested in proving that equation (17) for $E<\sqrt{V_{2}^{2}+V_{3}^{2}}$ still represents a real equation. Before we proceed with the proof, let us observe that

$$
z w=\exp \left[-2 i \arctan \frac{\sqrt{V_{2}^{2}+V_{3}^{2}-E^{2}}}{E}\right]
$$

and

$$
v_{ \pm}=\sqrt{\frac{2 m}{\hbar^{2}}}\left[\frac{1}{\sqrt{2}} \sqrt{V_{1}+\sqrt{V_{1}^{2}+V_{2}^{2}+V_{3}^{2}-E^{2}}} \pm i \frac{1}{\sqrt{2}} \sqrt{\sqrt{V_{1}^{2}+V_{2}^{2}+V_{3}^{2}-E^{2}}-V_{1}}\right] .
$$

It is natural to rewrite equation (17) as follows:

$$
\begin{aligned}
\tan (\epsilon a) & =-\epsilon \frac{\left(\nu_{+}-z w \nu_{-}\right) \tanh (\epsilon a)+(1-z w) \epsilon}{\nu_{-} \nu_{+}(1-z w) \tanh (\epsilon a)+\left(\nu_{-}-z w v_{+}\right) \epsilon} \\
& =-\epsilon \frac{\mathrm{NUM}}{\mathrm{DEN}}=-\epsilon \frac{\mathrm{NUM} \overline{\mathrm{DEN}}}{|\mathrm{DEN}|^{2}}
\end{aligned}
$$

The important point to note here is that

$$
\nu_{+}=\overline{\nu_{-}} \text {and }|z w|=1 \text {. }
$$


We can now proceed with the proof:

$$
\begin{aligned}
\mathrm{NUM} \overline{\mathrm{DEN}}= & {\left[\left(\nu_{+}-z w \nu_{-}\right) \tanh (\epsilon a)+(1-z w) \epsilon\right]\left[\left|\nu_{-}\right|^{2}(1-\overline{z w}) \tanh (\epsilon a)+\left(\nu_{+}-\overline{z w} \nu_{-}\right) \epsilon\right] } \\
= & \left(\nu_{-}-z w \nu_{-}+\overline{\nu_{-}}-\overline{z w} \overline{\nu_{-}}\right)\left|\nu_{-}\right|^{2} \tanh ^{2}(\epsilon a)+\left[v_{-}^{2}+{\overline{\nu_{-}}}^{2}-(z w+\overline{z w})\left|\nu_{-}\right|^{2}\right. \\
& \left.+(1-z w)(1-\overline{z w})\left|\nu_{-}\right|^{2}\right] \epsilon \tanh (\epsilon a)+\left(\nu_{-}-\overline{z w} \nu_{-}+\overline{\nu_{-}}-z w \overline{\nu_{-}}\right) \epsilon^{2} \\
& \in \mathbb{R} .
\end{aligned}
$$

\section{References}

[1] Adler S L 1995 Quaternionic Quantum Mechanics and Quantum Fields (New York: Oxford University Press)

[2] De Leo S and Scolarici G 2000 Right eigenvalue equation in quaternionic quantum mechanics J. Phys. A: Math. Gen. 33 2971-95

[3] De Leo S, Scolarici G and Solombrino L 2002 Quaternionic eigenvalue problem J. Math. Phys. 43 5815-29

[4] De Leo S and Ducati G 2001 Quaternionic differential operators J. Phys. Math. 42 2236-65

[5] Davies A J and McKellar B H 1989 Non-relativistic quaternionic quantum mechanics Phys. Rev. A 40 4209-14

[6] Davies A J and McKellar B H 1992 Observability of quaternionic quantum mechanics Phys. Rev. A 46 3671-75

[7] De Leo S, Ducati G and Nishi C 2002 Quaternionic potential in non-relativistic quantum mechanics J. Phys. A: Math. Gen. 35 5411-26

[8] Peres A 1979 Proposed test for complex versus quaternion quantum theory Phys. Rev. Lett. 42 683-6

[9] Kaiser H, George E A and Werner S A 1984 Neutron interferometric search for quaternions in quantum mechanics Phys. Rev. A $292276-9$

[10] Klein A G 1988 Schrödinger inviolate: neutron optical searches for violations of quantum mechanics $P$ hysica B 151 44-9

[11] De Leo S and Rodrigues W A 1997 Quantum mechanics: from complex to complexified quaternions Int. J. Theor. Phys. 36 1165-77

[12] De Leo S and Abdel-Khalek K 1997 Towards an octonionic world Int. J. Theor. Phys. 37 1945-85

[13] Rotelli P 1989 The Dirac equation on the quaternionic field Mod. Phys. Lett. A 4 933-40

[14] Conway A W 1937 Quaternion treatment of the relativistic wave equation Proc. Roy. Soc. A 162 145-54

[15] Hestenes D 1967 Real spinors field J. Math. Phys. 8 798-808

Hestenes D 1967 Spin and isospin J. Math. Phys. 8 809-12

[16] Gsponer A and Hurni J P 2001 Comment on formulating and generalizing Dirac's, Proca's, and Maxwell's equations with biquaternions or Clifford numbers Found. Phys. Lett. 14 77-85

[17] De Leo S 2001 Quaternionic Lorentz group and Dirac equation Found. Phys. Lett. 14 37-50

[18] De Leo S and Rotelli P 1996 Quaternionic electroweak theory J. Phys. G: Nucl. Part. Phys. 22 1137-50

[19] De Leo S and Ducati G 1999 Quanternionic group in physcis Int. J. Theor. Phys. 38 2197-220

[20] De Leo S and Ducati G 2003 Solving simple quaternionic differential equations J. Phys. Math. 44 2224-33

[21] Lévay P 1991 Quaternionic gauge fields and the geometric phase J. Math Phys. 32 2347-57

[22] De Leo S and Rotelli P 2002 Timelapse JETP Lett. 76 56-60

[23] De Leo S and Rotelli P 2004 Survival law in a potential model Phys. Rev. A 70 022101-11 